\title{
Is the pancreas affected in patients with septic shock? -- a prospective study
}

\author{
Raffaele Pezzilli, Alessandra Barassi, Andrea Imbrogno, Dario Fabbri, Antonina Pigna, \\ Antonio M. Morselli-Labate, Roberto Corinaldesi and Gianvico Melzi d'Eril
}

Bologna, Italy

BACKGROUND: Hyperamylasemia can be observed anecdotally during the course of severe sepsis or septic shock. This study aimed to investigate the possibility of pancreatic involvement in patients with septic shock using serum pancreatic enzyme determinations and imaging techniques in 21 consecutive patients with septic shock and 21 healthy subjects as controls.

METHODS: The serum activity of pancreatic amylase and lipase was assayed initially in all subjects and 24 and 48 hours after the initial observation in the 21 patients with septic shock. All patients also underwent radiological examination to detect pancreatic abnormalities.

RESULTS: The serum activity of pancreatic amylase was significantly higher in the 21 patients with septic shock than in the 21 control subjects during the study period, while the serum activity of lipase was similar to that of the control subjects. Amylase and lipase serum activity did not significantly changed throughout the study period in the 21 patients with septic shock. None of the patients with pancreatic hyperenzymemia had clinical signs or morphological alterations compatible with acute pancreatitis.

CONCLUSION: The presence of pancreatic hyperenzymemia in septic shock patients is not a biochemical manifestation of acute pancreatic damage, and the management of these patients should be dependent on the clinical situation and not merely the biochemical results.

(Hepatobiliary Pancreat Dis Int 2011; 10: 191-195)

Author Affiliations: Department of Digestive Diseases and Internal Medicine (Pezzilli R, Imbrogno A, Fabbri D, Morselli-Labate AM and Corinaldesi R), and Department of Surgery and Anesthesiology (Pigna A), Sant'OrsolaMalpighi Hospital, Alma Mater Studiorum University of Bologna, Bologna, Italy; Department of Medicine, Surgery and Odontoiatrics, University of Milan, Milan, Italy (Barassi A and Melzi d'Eril G)

Corresponding Author: Raffaele Pezzilli, MD, Dipartimento di Malattie Apparato Digerente e Medicina Interna, Ospedale Sant'Orsola-Malpighi, Via Massarenti 9, 40138 Bologna, Italy (Email: raffaele.pezzilli@aosp. bo.it)

(C) 2011, Hepatobiliary Pancreat Dis Int. All rights reserved.
KEY WORDS: amylase;

lipase;

clinical medicine;

shock;

sepsis;

systemic inflammatory response syndrome

\section{Introduction}

T $t$ has been reported that amylase seems to be more frequently elevated than lipase in patients with shock; however, none of these patients with pancreatic hyperenzymemia had pancreatic alterations on imaging. ${ }^{[1]}$ In addition, the occurrence of acute pancreatitis has rarely been reported after an episode of septic shock, ${ }^{[2,3]}$ and hyperamylasemia has also been observed anecdotally during the course of severe sepsis $^{[4]}$ or septic shock. ${ }^{[5]}$ In our previous study evaluating the frequency of pancreatic hyperenzymemia and acute pancreatitis, ${ }^{[1]}$ only 3 of 12 patients had septic shock. Thus we aimed to investigate the possibility of pancreatic involvement in patients with septic shock by serum pancreatic enzyme determinations and imaging techniques.

\section{Methods}

The study was carried out in the Intensive Care Unit (ICU) of Sant'Orsola-Malpighi Hospital (Bologna, Italy) and was approved by the local institutional review board with a priori patient or appropriate proxy consent obtained prior to the participants' entry into the study which was carried out in accordance with the Declaration of Helsinki.

The criteria for the presence of septic shock were the presence of an identifiable site of infection associated with the presence of a temperature greater than $38{ }^{\circ} \mathrm{C}$ or less than $36{ }^{\circ} \mathrm{C}$; a heart rate greater than 90 beats per minute; a respiratory rate of more than 20 breaths per 
minute; a white blood cell count $>12000$ or $<4000 / \mathrm{mm}^{3}$ and hypotension persisting despite fluid resuscitation and requiring vasopressor therapy. ${ }^{[6]}$ The exclusion criteria were patients under 18 years of age, the absence of circulating leukocytes, severely immunocompromised patients, autoimmune diseases, active chemotherapy or chronic steroid therapies. ${ }^{[7]}$ Acute pancreatitis was diagnosed on the basis of the presence of prolonged typical pancreatic pain associated with the findings of pancreatic abnormalities at imaging and a three-fold increase in the serum activity of amylase and lipase. ${ }^{[8]}$

From March 2009 to February 2010, 21 consecutive patients (13 males, 8 females; mean age 69.0 years, range 41-85 years) met these criteria and were enrolled in the study. All patients were treated according to current therapeutic modalities, ${ }^{[7]}$ and the final diagnoses were pneumonia in 5 patients, aortic prosthesis infection in 2, esophageal cancer in 2, gastric cancer in 2, pancreatic cancer in 2 , jejunal cancer in 1 , colon cancer in 1 , melanoma in 1 , chronic polyneuropathy in 1 , osteomyelitis in 1, upper gastrointestinal tract injury due to the ingestion of caustic substances in 1 , acute myocardial infarction in 1 , and infective endocarditis in the remaining patient.

Ten patients died during the hospital stay: 1 within 24 hours from ICU admission, 1 within 1 week from ICU admission, 4 between the 13th and the 18th day from ICU admission, 3 between the 31st and the 35th day from ICU admission and one 3 months after ICU admission.

Twenty-one subjects (11 males and 10 females, mean age 40.5 years, range $25-60$ years) recruited from blood donors, medical staff and subjects who underwent a routine medical check-up served as control subjects. They were in good general health, were not taking any medication, and had no signs of chronic or recent acute diseases.

Serum samples were obtained from all subjects at their initial observation and from the 21 patients with septic shock after 24 and 48 hours; they were kept frozen at $-20{ }^{\circ} \mathrm{C}$ until analysis.

Serum activity of pancreatic amylase (AMY-P, Roche, Milan, Italy; upper reference value, $115 \mathrm{U} / \mathrm{L}$ ) and lipase (LIPASE, Roche, Milan, Italy; upper reference value, 60 U/L) was assayed using commercially available kits. All patients also underwent ultrasonography and/or computed tomography to detect pancreatic abnormalities.

Data were expressed as mean \pm SD. Statistical analyses were carried out by the Mann-Whitney $U$ test, the matched-pairs Wilcoxon's test and the Spearman's rankorder correlation coefficient. The statistical analyses were performed by using the SPSS/PC+ statistical package
(SPSS Inc., Chicago, IL, USA) on a personal computer. Two-tailed $P$ values $<0.05$ were considered statistically significant.

\section{Results}

The time course of amylase and lipase is shown in Fig. 1. During the 3-day study period, the serum activity of pancreatic amylase was significantly higher in the patients with septic shock than in the controls. On the contrary, the serum activity of lipase was similar to that of the control subjects. When only patients with septic shock were considered, we found that the serum activity of amylase and lipase did not change throughout the study period.

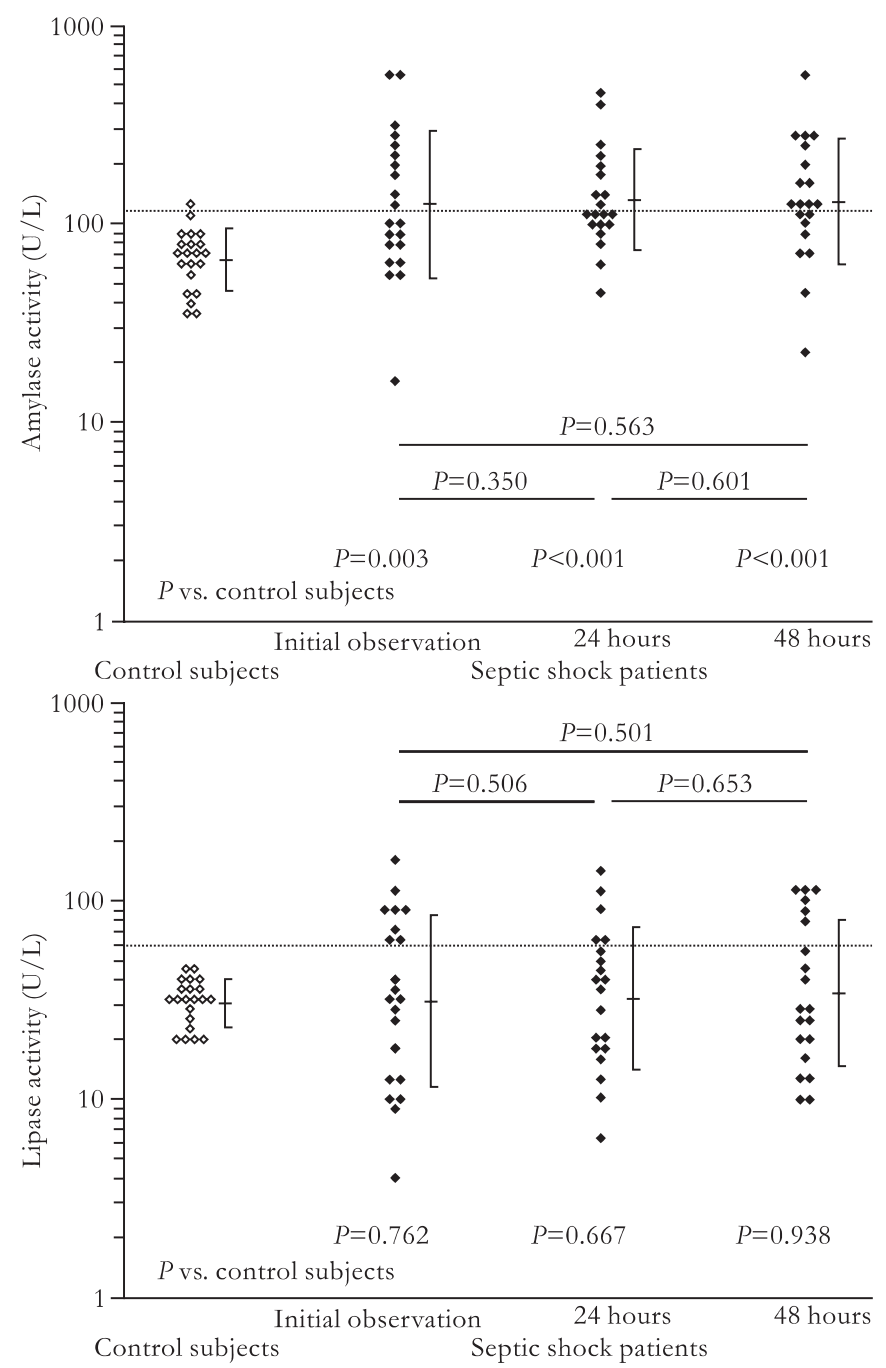

Fig. 1. Individual serum activity of pancreatic amylase and lipase in patients with septic shock during the study period and in the control subjects. Data are reported as mean \pm SD. Horizontal dotted lines indicate the upper reference limit of each enzyme. 
Table. Pancreatic amylase and lipase serum activity in survivors and non-survivors throughout the study period $(\mathrm{U} / \mathrm{L}$, mean $\pm \mathrm{SD})$

\begin{tabular}{|c|c|c|c|}
\hline & Survivors $(n=11)$ & Non-survivors $(n=10)$ & $P$ value \\
\hline \multicolumn{4}{|l|}{ Amylase } \\
\hline Initial observation & $127 \pm 65$ & $226 \pm 212$ & 0.756 \\
\hline 24 hours & $139 \pm 54$ & $176 \pm 148$ & 0.603 \\
\hline 48 hours & $156 \pm 84$ & $171 \pm 155$ & 0.941 \\
\hline \multicolumn{4}{|l|}{ Lipase } \\
\hline Initial observation & $41 \pm 29$ & $54 \pm 51$ & 0.973 \\
\hline 24 hours & $47 \pm 37$ & $39 \pm 32$ & 0.503 \\
\hline 48 hours & $55 \pm 40$ & $37 \pm 34$ & 0.261 \\
\hline
\end{tabular}

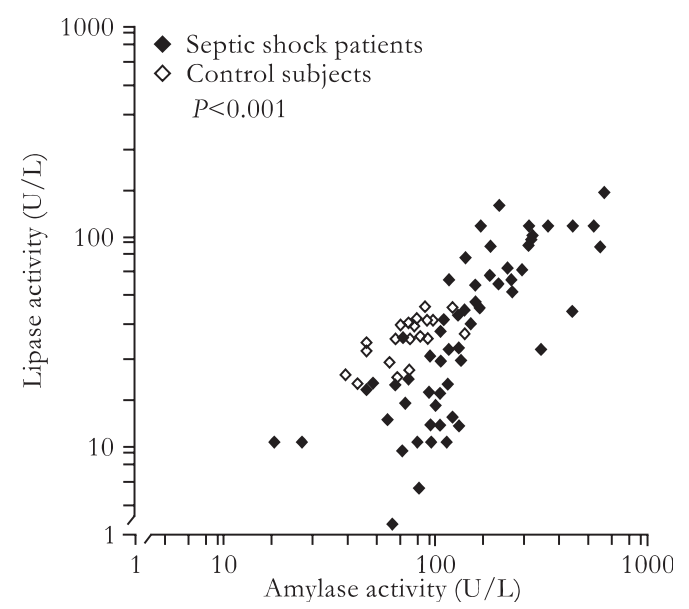

Fig. 2. Relationship between serum amylase and lipase activity in the 83 determinations performed on the 21 patients with septic shock followed for 3 days, and in the 21 control subjects.

Two patients (9.5\%), one with upper gastrointestinal tract injury due to the ingestion of causative substances and the other with infection of an aortic prosthesis, had serum activity of pancreatic amylase three times greater than the upper reference limit while none of the patients studied had serum lipase activity three times greater than the upper reference limit. Considering the patients who died during hospitalization and survivors, we did not find significant differences in the serum activity of amylase and lipase between the survivors and nonsurvivors in the 3 days of the study (Table).

Pooling all the data obtained at the different time intervals, a significant relationship was found between serum amylase and lipase concentrations $(P<0.001)$ (Fig. $2)$. This relationship persisted when the determinations made in the patients and control subjects $(P<0.001$ for both) were considered separately.

Finally, none of the patients developed clinical signs or morphological changes compatible with those of acute pancreatitis.

\section{Discussion}

One of the consequences of septic shock is ischemia, and the ischemia followed by reperfusion results in a breakdown of the microcirculation in the pancreas, which occurs as in other organs, and this is considered to be a critical factor in the pathogenesis of acute pancreatitis. ${ }^{[9,10]}$ In addition, microvascular perfusion failure is a characteristic hallmark of pancreatitis. ${ }^{[10-12]}$ Since Panum ${ }^{[13]}$ presented the first report, other researchers have reported that the pancreas is highly sensitive to ischemia. ${ }^{[14-18]}$ In humans, acute pancreatitis may develop after embolic closure of the vessels supplying the pancreas, ${ }^{[19]}$ after hypoperfusion of the pancreas during cardiac surgery $^{[20,21]}$ and surgery for thoraco-abdominal aneurysms, ${ }^{[22]}$ after splanchnic hypoperfusion, ${ }^{[23]}$ or after pancreatic transplantation. ${ }^{[24]}$ We found that during 12 hours after admission, serum amylase concentrations were elevated, whereas serum lipase concentrations were below the upper reference limit, suggesting that hyperamylasemia is of non-pancreatic origin. ${ }^{[1]}$ Hegewald et $\mathrm{al}^{[25]}$ and Gmaz-Nikulin et $\mathrm{al}^{[23]}$ reported the appearance of pancreatic changes during shock, detected only by electron microscopy and histology; in our study, we used imaging techniques, such as ultrasonography and computed tomography, in evaluating the changes of the pancreatic gland, and the pancreatic changes were not clinically relevant. Interestingly, hyperamylasemia was also observed anecdotally in the course of severe sepsis ${ }^{[4]}$ or septic shock. ${ }^{[5]}$ Previously we evaluated the frequency of pancreatic hyperenzymemia and acute pancreatitis, ${ }^{[1]}$ and we found that only 3 of 12 patients had septic shock. Thus investigated in the present study the possibility of pancreatic involvement in patients with septic shock using serum pancreatic enzyme determinations and imaging techniques. As pointed out by Rogers, ${ }^{[5]}$ biochemical investigation is no substitute for clinical examination. In fact, even if the serum activity of pancreatic amylase was significantly higher in patients with septic shock than in control subjects, the serum activity of lipase was similar to that of the control subjects; none of the patients developed clinical signs or morphological alterations compatible with those of acute pancreatitis. Thus, other causes should be hypothesized for the increased level of serum amylase. For example, in the present study, 7 patients had gastrointestinal tract diseases and 2 were operated on for a pancreatic neoplasm (9/21, 42.9\%). Although pancreatic hyperenzymemia was observed in these patients, who were free from any kind of contemporary shock condition, we believe that this evidence does not affect our data because only a low percentage of the patients (9.5\%) had a serum activity of pancreatic amylase three times greater than the upper 
reference limit i.e. one patient had upper gastrointestinal tract injury due to the ingestion of causative substances, and the other had an infection of an aortic prosthesis. It is well-known that hyperamylasemia may be observed in acute abdominal conditions other than acute pancreatitis such as acute peritonitis, acute appendicitis, renal calculi and intestinal obstruction, and abdominal surgery. ${ }^{[26-31]}$ However, this was not the case in our patients. Our data also agree with those of Vadas et $\mathrm{al}^{[32]}$ who found similar data in septic shock patients by using phospholipase A2 determination. These authors reported that their data on phospholipase A2 levels were consistent with an extrapancreatic source of intravascular phospholipase A2 release during sepsis and these levels correlated directly with the magnitude and duration of circulatory collapse, with a progressive fall of serum PLA2 levels during convalescence.

Finally, none of the patients with septic shock had serum lipase activity three times greater than the upper reference limit. This further confirmed that serum lipase is more accurate than pancreatic amylase in the diagnosis of acute pancreatitis. ${ }^{[33,34]}$ It has also been reported that sepsis is associated with secretory pancreatic dysfunction that is worse in septic shock patients than in sepsis patients without shock and that impaired exocrine function is significantly correlated to APACHE III and SOFA scores. ${ }^{[35]}$ However, these findings do not influence the result of our study because we aimed to evaluate the presence of acute pancreatic alterations in patients with septic shock.

Considering patients who died during hospitalization and survivors, we found no differences in the serum activity of amylase and lipase between the survivors and non-survivors in the 3 days of the study.

In conclusion, during the early phases of septic shock, serum amylase is more frequently elevated than serum lipase, but none of the patients with pancreatic hyperenzymemia showed pancreatic injury at imaging. The presence of hyperamylasemia in a patient with septic shock is not a biochemical manifestation of acute pancreatic damage and the management of the patient should depend on clinical conditions and not merely on evaluation of biochemical results.

\section{Funding: None.}

Ethical approval: Not needed.

Contributors: PR and PA proposed the study. PR wrote the first draft. IA, FD and MLAM analyzed the data. All authors contributed to the design and interpretation of the study and to further drafts. $\mathrm{PR}$ is the guarantor.

Competing interest: No benefits in any form have been received or will be received from a commercial party related directly or indirectly to the subject of this article.

\section{References}

1 Pezzilli R, Morselli-Labate AM, Romboli E, Dibenedetti F, Massa M, Migliori M, et al. Pancreatic involvement during the early phase of shock. JOP 2002;3:139-143.

2 Chiu CH, Lin TY, Wu JL. Acute pancreatitis associated with streptococcal toxic shock syndrome. Clin Infect Dis 1996;22: 724-726.

3 Adams D, Fenton SJ, Nichol PF. Streptococcal pancreatitis and toxic shock syndrome in a 2-month-old infant. J Pediatr Surg 2007;42:261-263.

4 Frulloni L, Patrizi F, Bernardoni L, Cavallini G. Pancreatic hyperenzymemia: clinical significance and diagnostic approach. JOP 2005;6:536-551.

5 Rogers KL, Date RS, Ward JB. Biochemical investigation is no substitute for clinical examination! JOP 2008;9:209-211.

6 Bone RC. Toward an epidemiology and natural history of SIRS (systemic inflammatory response syndrome). JAMA 1992;268:3452-3455.

7 Iapichino G, Marzorati S, Umbrello M, Baccalini R, Barassi $A$, Cainarca $M$, et al. Daily monitoring of biomarkers of sepsis in complicated long-term ICU-patients: can it support treatment decisions? Minerva Anestesiol 2010;76:814-823.

8 Pezzilli R, Venturi M, Morselli-Labate AM, Ceciliato R, Lamparelli MG, Rossi A, et al. Serum trypsinogen activation peptide in the assessment of the diagnosis and severity of acute pancreatic damage: a pilot study using a new determination technique. Pancreas 2004;29:298-305.

9 Hoffmann TF, Leiderer R, Waldner H, Arbogast S, Messmer $K$. Ischemia reperfusion of the pancreas: a new in vivo model for acute pancreatitis in rats. Res Exp Med (Berl) 1995;195: 125-144.

10 Klar E, Endrich B, Messmer K. Microcirculation of the pancreas. A quantitative study of physiology and changes in pancreatitis. Int J Microcirc Clin Exp 1990;9:85-101.

11 Klar E, Messmer K, Warshaw AL, Herfarth C. Pancreatic ischaemia in experimental acute pancreatitis: mechanism, significance and therapy. Br J Surg 1990;77:1205-1210.

12 Kusterer K, Enghofer M, Zendler S, Blochle C, Usadel KH. Microcirculatory changes in sodium taurocholate-induced pancreatitis in rats. Am J Physiol 1991;260:G346-351.

13 Panum P. Experimental contributions to the theory of embolism. Virkows Arch 1886;25:308-338.

14 Spormann H, Sokolowski A, Letko G. Effect of temporary ischemia upon development and histological patterns of acute pancreatitis in the rat. Pathol Res Pract 1989;184:507-513.

15 Pfeffer RB, Lazzarini-Robertson A Jr, Safadi D, Mixter G Jr, Secoy CF, Hinton JW. Gradations of pancreatitis, edematous, through hemorrhagic, experimentally produced by controlled injection of microspheres into blood vessels in dogs. Surgery 1962;51:764-769.

16 Redha F, Uhlschmid G, Ammann RW, Freiburghaus AU. Injection of microspheres into pancreatic arteries causes acute hemorrhagic pancreatitis in the rat: a new animal model. Pancreas 1990;5:188-193.

17 Fleischer GM, Herden P, Spormann H. Animal studies on the role of ischemia in the pathogenesis of acute pancreatitis. $\mathrm{Z}$ Exp Chir Transplant Kunstliche Organe 1984;17:179-187.

18 Sokolowski A, Spormann H, Urbahn H, Letko G. Contribution of pancreatic edema and short-term ischemia to experimental acute pancreatitis in the rat. II. Behaviour of 
serum parameters. Z Exp Chir Transplant Kunstliche Organe 1986;19:331-339.

19 Probstein JC, Joshi RA, Blumenthal HT. Atheromatous embolization; an etiology of acute pancreatitis. AMA Arch Surg 1957;75:566-572.

20 Fernández-del Castillo C, Harringer W, Warshaw AL, Vlahakes GJ, Koski G, Zaslavsky AM, et al. Risk factors for pancreatic cellular injury after cardiopulmonary bypass. $\mathrm{N}$ Engl J Med 1991;325:382-387.

21 Warshaw AL, O'Hara PJ. Susceptibility of the pancreas to ischemic injury in shock. Ann Surg 1978;188:197-201.

22 Gullo L, Cavicchi L, Tomassetti P, Spagnolo C, Freyrie A, D'Addato M. Effects of ischemia on the human pancreas. Gastroenterology 1996;111:1033-1038.

23 Gmaz-Nikulin E, Nikulin A, Plamenac P, Hegewald G, Gaon D. Pancreatic lesions in shock and their significance. J Pathol 1981;135:223-236.

24 Sakorafas GH, Tsiotos GG, Sarr MG. Ischemia/ReperfusionInduced pancreatitis. Dig Surg 2000;17:3-14.

25 Hegewald G, Nikulin A, Gmaz-Nikulin E, Plamenac P, Barenwald G. Ultrastructural changes of the human pancreas in acute shock. Pathol Res Pract 1985;179:610-615.

26 Chase CW, Barker DE, Russell WL, Burns RP. Serum amylase and lipase in the evaluation of acute abdominal pain. Am Surg 1996;62:1028-1033.

27 Pezzilli R, Ventrucci M, Billi P, Conci T, Fontana G. Behaviour of serum pancreatic enzymes in intestinal occlusion. Ital J Gastroenterol 1991;23:166.

28 Apostolov I, Minkov N, Koycheva M, Isterkov M, Abadjyev $\mathrm{M}$, Ondeva $\mathrm{V}$, et al. Acute changes of serum markers for tissue damage after ESWL of kidney stones. Int Urol Nephrol 1991;23:215-220.

29 Swensson EE, Maull KI. Clinical significance of elevated serum and urine amylase levels in patients with appendicitis. Am J Surg 1981;142:667-670.

30 Baniel J, Leibovitch I, Foster RS, Rowland RG, Bihrle R, Donohue JP. Hyperamylasemia after post-chemotherapy retroperitoneal lymph node dissection for testis cancer. J Urol 1995;154:1373-1375.

31 Miyagawa S, Makuuchi M, Kawasaki S, Kakazu T, Hayashi K, Kasai H. Serum Amylase elevation following hepatic resection in patients with chronic liver disease. Am J Surg 1996;171:235-238.

32 Vadas P, Pruzanski W, Stefanski E, Sternby B, Mustard R, Bohnen J, et al. Pathogenesis of hypotension in septic shock: correlation of circulating phospholipase A2 levels with circulatory collapse. Crit Care Med 1988;16:1-7.

33 Gumaste VV, Roditis N, Mehta D, Dave PB. Serum lipase levels in nonpancreatic abdominal pain versus acute pancreatitis. Am J Gastroenterol 1993;88:2051-2055.

34 Pezzilli R, Billi P, Fiocchi M, Ossani M, Sprovieri G, Fontana G. Serum lipase assay. A test of choice in acute pancreatitis. Panminerva Med 1992;34:30-34.

35 Tribl B, Sibbald WJ, Vogelsang H, Spitzauer S, Gangl A, Madl C. Exocrine pancreatic dysfunction in sepsis. Eur J Clin Invest 2003;33:239-243.

Received December 7, 2010

Accepted after revision January 21, 2011 